\title{
Revised Force Control Using a Compliant Sensor with a Position Controlled Robot
}

\author{
Friedrich Lange, Claudius Jehle, Michael Suppa, and Gerd Hirzinger
}

\begin{abstract}
A different way of force control is presented, that is especially advantageous for position controlled robots. Instead of usual force control laws we rely on the well tuned position control loop and just use the force sensor to measure the target pose or to predict the desired trajectory. In combination with a compliant sensor we introduce an inherently stable framework of force control which almost inhibits all control errors. After an unexpected impact the force error is reduced independently from the sensor's bandwidth or delays in signal processing. Thus the (inevitable) impact force is more significant than the measured force control errors. The special case of a sensor that is mounted far away from a vertex-face contact is discussed, too.
\end{abstract}

\section{INTRODUCTION}

Force control has been discussed and implemented by universities for more than 20 years using different types of sensors and different control strategies. So what is the motivation to reconsider the topic again?

There are still limitations, e.g. when striking an unexpected object. And the general purpose force control in all 6 degrees-of-freedom (dof) is limited to the case in which the robot and its environment are rigidly connected. Otherwise the robot may rotate because it senses a moment though at the contact point (tool center point, tcp) there is only a force.

Both problems are addressed in this paper. In addition, a force sensor is used in a similar manner as a position sensor. This facilitates programming since positions and orientations are more intuitive than forces and moments.

The first basic idea is that a force sensor has to be compliant. Compliant (elastic) force sensors differ from stiff sensors, as they do not use high-sensitive strain gauge elements. A compliant force sensor is available by SCHUNK ${ }^{\circledR}$ [1], [2]. It is realized by position sensitive devices (PSD) to measure the deflections of springs within the sensor. Typical deflections of this sensor are $1 \mathrm{~mm}$ or $1 \mathrm{deg}$, corresponding to forces and moments of $300 \mathrm{~N}$ or $7 \mathrm{Nm}$, where the latter are scalable [3] depending on the application. Thus with a compliant sensor, high bandwidth positional disturbances cause deflections, but not necessarily significant forces or moments. Therefore such a sensor is fundamental for many tasks, e.g. in moving belt production as in [3] (see Fig. 1). Especially when the end-effector is connected to an object that is rigidly attached to the conveyor, shaking of the

The authors are with the German Aerospace Center (DLR), Robotics and Mechatronics Center (RMC), D-82234 Wessling, Germany friedrich.langeddlr.de

This work is partially funded by KUKA Laboratories GmbH. The authors want to thank Johannes Scharrer, Rüdiger Spillner, and Yi Shen from the Technical University of Munich (Institute for Machine Tools and Industrial Management $i w b$ ) and Simon Kriegel (RMC) for their support. conveyor belt would cause intolerable forces if there is no compliance. Besides, a compliant sensor is necessary when approaching to unknown objects, as long as no non-contact sensor ([4], [5], [6]) is provided, that can predict the position of the impact. The drawback of compliant sensors is the possibility of oscillations of the compliance when no contact is present. This problem has been addressed in [7].

A compliant force/torque sensor may be considered as a force measuring device or as a position sensor. The latter interpretation is advantageous with position controlled robots, since e.g. the compensation of a deflection of $0.5 \mathrm{~mm}$ is simply done by moving the robot for $0.5 \mathrm{~mm}$. Available sensors [2] support the mode to output the deflections directly, instead of forces and moments. Thus force control with small forces and moments is similar to position control. So a compliant force sensor might be used in the same way as e.g. a laser distance sensor.

The second basic idea is obvious. For force control the existing position control loop may be used instead of creating an extra force control loop. Position control is typical for industrial robots. So no additional controller has to be designed or tuned.

Thus the question of the first paragraph can be answered: The two basic ideas allow fast and simple force control. Fast, since the reaction to unexpected forces is not affected by a slow force control loop. And simple, since programming only deals with position and orientation. This allows flexible solutions, e.g. the approach speed can be chosen independently from a desired force after contact. Thus sensitive objects can

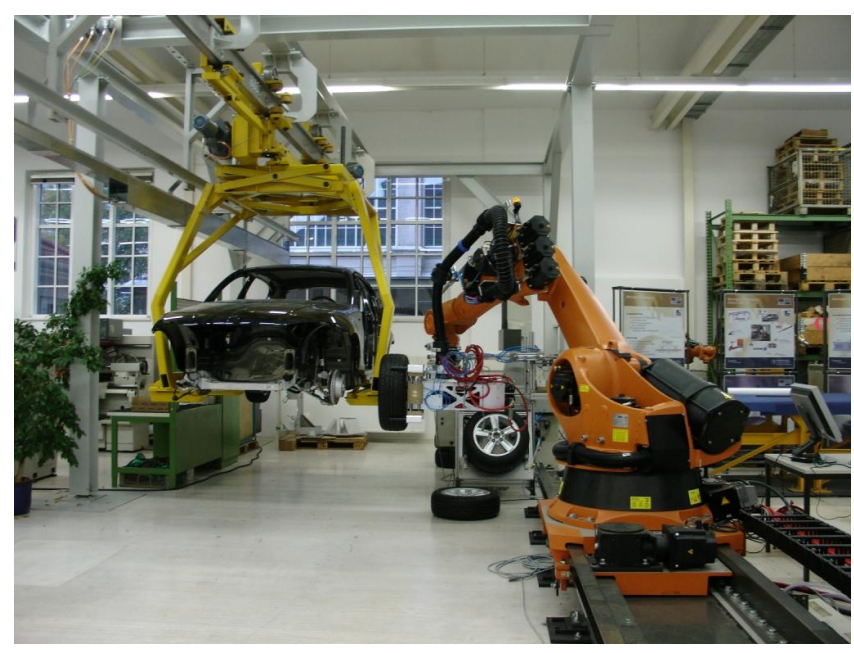

Fig. 1. Set-up at $i w b$ for mounting wheels to a moving car body. 


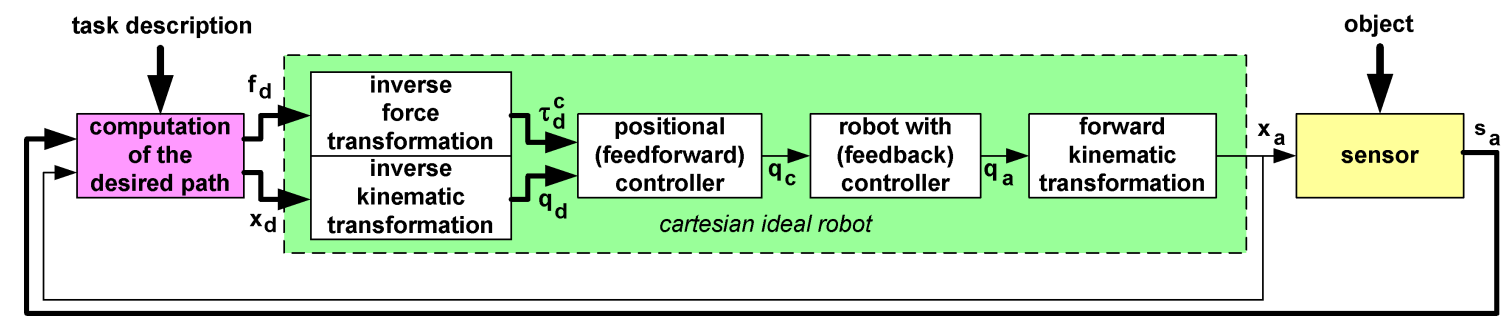

Fig. 2. Control architecture with position control as inner loop (green) and the computation of the desired path from sensor values $\mathbf{s}_{a}$ as outer loop

be handled. In addition, assembly tasks can be formulated easier, as usually a position and a tolerance is given, but no contact force. Assembly will succeed if chamfers are present and the tolerance is less than the allowed deflection of the sensor.

It should be mentioned that at the time instant of an impact not only the measured forces and torques are present but also an impact force which is caused by a strong deceleration. With a compliant sensor this force, which can hardly be reduced by control or by a compliant design, may be dominant with respect to the measured force. So it represents a limit for the performance of force control. This limit will be estimated.

The rest of the paper is organized as follows: Section II first reviews the standard force control scheme for position controlled robots. Then the proposed approach is presented and the differences are discussed. Section III shows an extension, in order to prevent an orientational drift that would be caused by a sensor that is mounted distantly from the contact point. Section IV appraises the impact when approaching to unknown objects. Finally, experiments are presented and a conclusion is given. In addition, a video clip is attached that illustrates the results.

\section{Force Control with Position Controlled Roвотs}

This section first reviews the well known inner-loop outerloop force control approach that is used with industrial robots. Then the proposed method is explained. This method basically reduces force errors. But it is possible to use it with an impedance-based task specification that smoothes the robot motion.

\section{A. Standard Approach}

[8] outlines different force control schemes. Since with industrial robots there is no torque interface available, position based control is required. This means that a force control loop is closed around the existing position control loop. This is called indirect control or, more specifically, admittance control.

The typical force control law of industrial robots is represented by

$$
\mathbf{x}_{c}(k)=\mathbf{x}_{c}(k-1)+\mathbf{K} \cdot\left(\mathbf{f}(k)-\mathbf{f}_{d}\right)+\mathbf{D} \cdot(\mathbf{f}(k)-\mathbf{f}(k-1))
$$

where $\mathbf{x}$ and $\mathbf{f}$ are Cartesian $6 \times 1$ vectors of the pose (position and orientation) and of forces and moments re- spectively ${ }^{1} \cdot \mathbf{x}_{c}$ is the commanded pose which, transformed to the robot joint space, acts as the desired value of the position control loops. In contrast, the term desired pose is used within this paper as the pose at which the desired forces and moments are reached with the given environment. $k$ represents the index of the sampling step. $\mathbf{f}_{d}$ is the vector of the desired forces and moments, which are assumed constant. $\mathbf{K}$ and $\mathbf{D}$ are the $6 \times 6$ diagonal matrices of the controller parameters. In the following, this control approach is referred to as a pd-controller.

The position control loop depends on the robot manufacturer.

\section{B. Proposed Approach}

In contrast to the method of (1), in this section a desired pose is computed by a force control law and regulated by advanced position control (see Fig. 2). The desired pose $\mathbf{x}_{d}(k)$ of the current time step is computed by

$$
\mathbf{x}_{d}(k)=\mathbf{x}_{a}(k)+\mathbf{C} \cdot\left(\mathbf{f}(k)-\mathbf{f}_{d}\right)
$$

from the current force error and the current actual pose $\mathbf{x}_{a}(k)$. The use of an actual (=measured by the motor encoders) pose $\mathbf{x}_{a}(k)$ instead of the previous commanded pose $\mathbf{x}_{c}(k-1)$ gives a fundamental difference. Former robot interfaces did not provide the actual pose. Therefore (2) was not realizable. Since today's robots have this feature, force control can profit from it.

Thus, strictly speaking, (2) is no control law but a geometric task description of the desired pose. It should be mentioned that (2) is not depending on other time steps but valid for all time instants. This allows to compute the desired pose from the measurements of a single time step with contact. $\mathbf{C}$ is then no controller design parameter but the compliance matrix of the sensor, i.e. the inverse stiffness matrix. Implicitly it is assumed that the forces and moments are measured synchronously to the robot's actual pose.

Besides, the position controlled robot is not only feedbackcontrolled. ${ }^{2}$ Instead, it is assumed to be ideal which means that the actual pose reaches the desired pose without any control error or time delay. This requires a feedforward controller which in addition processes future desired poses, not only the current values. This is especially useful for robot trajectories with a priori known accelerations. Furthermore, as

\footnotetext{
${ }^{1}$ In some implementations the sign of the forces and moments is different.

${ }^{2}$ This feature is advantageous but not mandatory for the proposed approach.
} 


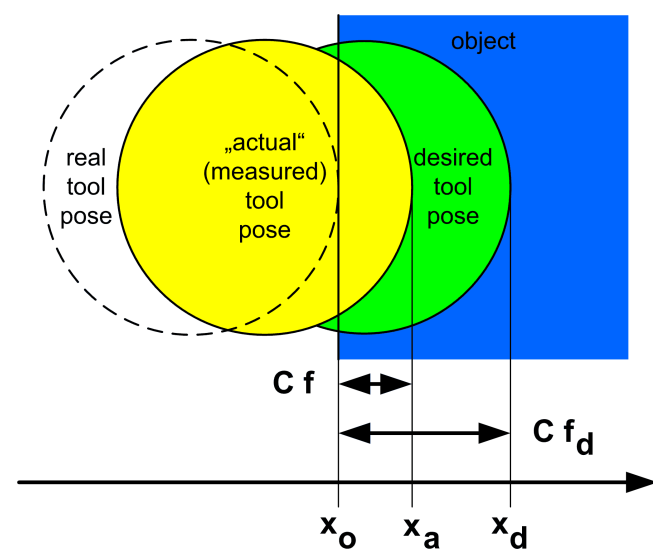

Fig. 3. Notation when pressing a pin against an object.

a disturbance variable compensation, the desired forces and moments are considered. Nevertheless, unexpected sensor values that detect a contact state deteriorate the performance since then the previously predicted future values are not correct. In contrast, an expected impact can be taken into account.

For convenience the position control is implemented in joint space, meaning that commanded joint angles $\mathbf{q}_{c}(k)$ are sent to the internal feedback controllers instead of a Cartesian vector $\mathbf{x}_{c}(k)$.

The contact is modeled according to Fig. 3. The object is assumed to be rigid while the sensor is compliant. Thus the actual pose of the robot represents a virtual pose $\mathbf{x}_{a}$ of the tcp. The real pose of the tcp is constant and coincides with the object pose $\mathbf{x}_{o} \cdot{ }^{3}$ Forces and moments are generated by the displacement of the real tcp with respect to the virtual one, where all deflections are assumed to be within the sensor.

\section{Discussion}

Equation (1) is unfavorable if the robot approaches to an object of unknown pose since during the free motion the commanded pose is advanced with respect to the actual pose $\left(\mathbf{x}_{c}(k)>\mathbf{x}_{c}(k-1)>\mathbf{x}_{c}(k-2)>\cdots \Rightarrow \mathbf{x}_{c}(k) \gg \mathbf{x}_{a}(k)\right)$. Thus, when the contact is reached, the commanded pose has not only to be modified by the current control difference but in addition by the delay due to the robot dynamics. For stability reasons this takes a large number of sampling steps.

In contrast, (2) allows an instantaneous computation of the desired pose. The sum of the sensor values $\mathbf{s}_{a}=\mathbf{C} \cdot \mathbf{f}$ and the actual robot pose $\mathbf{x}_{a}$ is not interpreted as a feedback of the robot pose but as the object pose $\mathbf{x}_{o}$.

This means that control according to Fig. 2, using (2), cannot become unstable. The dynamics are given exclusively by the position control. Stability is guaranteed by the positional feedback controller, since the feedforward part has no influence on stability.

\footnotetext{
${ }^{3}$ The coordinate systems are aligned to the surfaces of pin and object. Alternatively a known offset has to be incorporated.
}

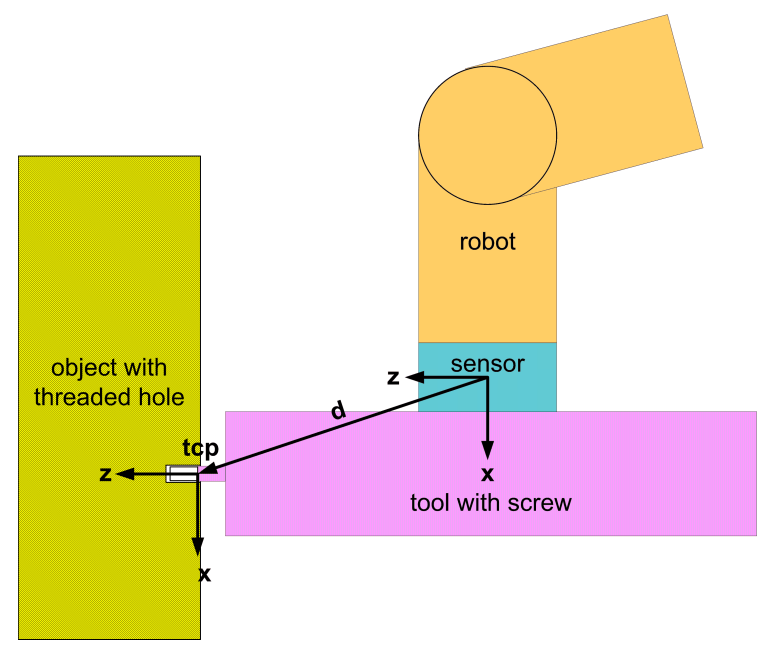

Fig. 4. Nominal setup with a tool that has to press against the object, in order to insert a screw or a pin.

This is valid as long as $\mathbf{C}$ represents the compliance of the system correctly. Otherwise there are couplings between the computed object pose and the robot motion, which finally may cause instability if $\mathbf{C}$ is assumed much bigger than real.

It should be noted that the position feedback is typically sampled at a higher rate than the force control interface. This is of use for the proposed approach, since for a time-invariant set-up the desired pose is constant.

\section{Force CONTRol With a Distant SEnsor}

So far the method is close to [3]. It works well, if the sensor is close to the contact between the robot tool and the object. It still works if the sensor is located on the force vector which is a line through the contact point, usually perpendicular to the object surface. But the force sensor is typically mounted between the robot and the tool (see Fig. 4). Thus a moment in the sensor is measured, that will cause a rotation of the robot tool, though at the tool center point (tcp) there is no moment at all.

This is extremely disadvantageous if the contact has a vertex-face topology, since then the rotation will not cause a restoring moment. Therefore, previous work tried to identify the topology, see [9], [10], [3] for more information. In contrast, here a generic approach is presented which allows force control with a distant sensor, without generating unwanted forces or moments at the tcp. For the example of Fig. 4 it generates a rotation, such that the desired forces are present at the tcp and at the same time the desired orientation of the tcp is maintained. This is achieved by a posture as in Fig. 5.

\section{A. Transformations}

At the sensor, $3 \times 1$ forces $\mathbf{f}_{s}$ and moments $\mathbf{m}_{s}$ cause deflections ${ }^{s} \mathbf{x}_{s^{\prime}}$ between the nominal sensor frame $s$ and the deflected sensor frame $s^{\prime}{ }^{4}$. When considering $3 \times 1$ vectors

\footnotetext{
${ }^{4}$ The notation ${ }^{a} \mathbf{x}_{b}$ represents a general transformation from frame a to frame $b$, expressed in frame a. The rotation from a nominal to a deflected frame is small enough, so that no singularities of the notation have to be considered.
} 


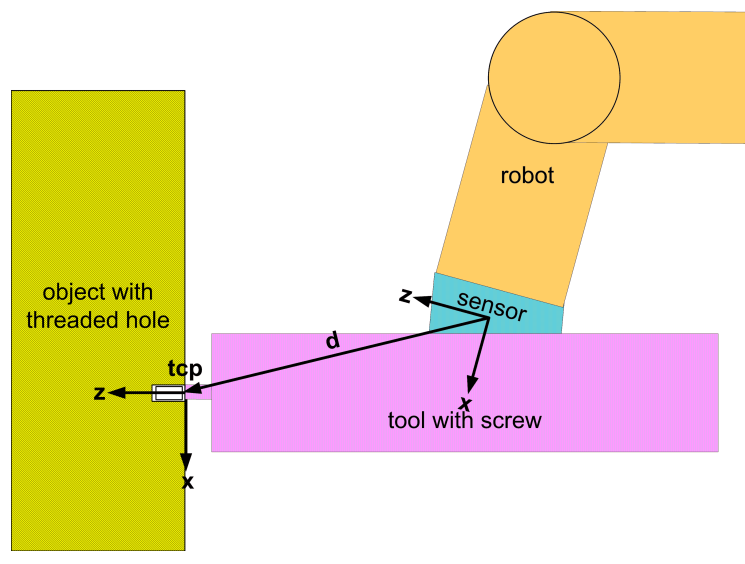

Fig. 5. Desired posture with nominal orientation of the screw.

of Cartesian positional deflection ${ }^{s} \mathbf{p}_{s^{\prime}}$ and orientational deflection ${ }^{s} \varphi_{s^{\prime}}$, the following equations hold

$$
\begin{aligned}
{ }^{s} \mathbf{p}_{s^{\prime}} & =\mathbf{C}_{p} \cdot \mathbf{f}_{s} \\
{ }^{s} \varphi_{s^{\prime}} & =\mathbf{C}_{\varphi} \cdot \mathbf{m}_{s},
\end{aligned}
$$

where $\mathbf{C}_{p}$ and $\mathbf{C}_{\varphi}$ are the $3 \times 3$ positional and orientational compliance matrices which are a characteristic of the sensor.

The forces and moments at the sensor may be transformed to the tcp, using the well known wrench equations

$$
\begin{aligned}
\mathbf{f}_{s} & =\mathbf{f}_{t} \\
\mathbf{m}_{s} & =\mathbf{m}_{t}+\mathbf{d} \times \mathbf{f}_{t},
\end{aligned}
$$

where the index ${ }_{t}$ stands for the tcp and $\mathbf{d}$ is the vector from the sensor to the tcp.

On the other hand, the Cartesian deflection is transformed using the twist equations 5

$$
\begin{aligned}
{ }^{s} \mathbf{p}_{s^{\prime}} & ={ }^{t} \mathbf{p}_{t^{\prime}}+\mathbf{d} \times{ }^{t} \varphi_{t^{\prime}} \\
{ }^{s} \varphi_{s^{\prime}} & ={ }^{t} \varphi_{t^{\prime}},
\end{aligned}
$$

where ${ }^{t} \mathbf{p}_{t^{\prime}}$ and ${ }^{t} \varphi_{t^{\prime}}$ are the positional and orientational deflections at the tcp.

\section{B. Reformulation of the force control task}

These equations allow the computation of the desired deflections ${ }^{t d} \mathbf{p}_{t d^{\prime}}$ and ${ }^{t d} \varphi_{t d^{\prime}}$ at the tcp, if the desired forces $\mathbf{f}_{t d}$ and moments $\mathbf{m}_{t d}$ at the tcp are given:

$$
\begin{aligned}
{ }^{t d} \mathbf{p}_{t d^{\prime}} & =\mathbf{C}_{p} \cdot \mathbf{f}_{t d}-\mathbf{d} \times\left(\mathbf{C}_{\varphi} \cdot\left(\mathbf{m}_{t d}+\mathbf{d} \times \mathbf{f}_{t d}\right)\right) \\
{ }^{t d} \varphi_{t d^{\prime}} & =\mathbf{C}_{\varphi} \cdot\left(\mathbf{m}_{t d}+\mathbf{d} \times \mathbf{f}_{t d}\right) .
\end{aligned}
$$

Thus, control is to move the robot in such a way that the desired deflections are measured. Since the robot is controlled in a reference system $r$ which is close to the tcp frame, this is accomplished similar to (2) by

$$
{ }^{r} \mathbf{x}_{t d}(k)={ }^{r} \mathbf{x}_{t a}(k)+{ }^{t a} \mathbf{x}_{t a^{\prime}}-{ }^{t d} \mathbf{x}_{t d^{\prime}}
$$

\footnotetext{
${ }^{5}$ The twist equations are an approximation of the product of homogeneous transformation matrices ${ }^{s} \mathbf{T}_{s^{\prime}}={ }^{s} \mathbf{T}_{t} \cdot{ }^{t} \mathbf{T}_{t^{\prime}} \cdot{ }^{s} \mathbf{T}_{t}-1$, which is a more accurate solution. ${ }^{s} \mathbf{T}_{t}={ }^{s /} \mathbf{T}_{t \prime}$ is the generic representation of the vector d. Without loss of generality, in this paper it is assumed that the nominal sensor frame and the nominal tcp frame are parallel.
}

where the indices $t a$ and $t a^{\prime}$ stand for the nominal and the deflected actual pose of the tcp. ${ }^{6} 7$

$$
{ }^{r} \mathbf{x}_{o}={ }^{r} \mathbf{x}_{t a}(k)+{ }^{t a} \mathbf{x}_{t a^{\prime}}=\mathbf{x}_{t a^{\prime}}=\mathbf{x}_{t d^{\prime}}
$$

is called the object position. This is the surface of the object at which the deflected pose is aligned both, in the actual and the desired state.

Equations (11) and (12) assume that there is no compliance besides the sensor. If instead there is significant compliance in the robot joints or in the object pose, the equations have to be adapted.

\section{ASSESSMENT OF THE IMPACT}

So far, measured forces and moments are controlled. Additional forces arise when the robot tool touches an object, due to the deceleration of the tool. In Fig. 3, during the contact the real tool position has been assumed to be identical with the object surface. In reality, at the short instant of impact, the tool will slightly indent into the surface, yielding an additional resetting contact force, the impact force. This problem has been ignored in robotics since this force is not measured by a robot mounted sensor and thus it has no effect on force control. In addition, the impact force acts on the robot dynamics only as a missing deceleration force. However, an impact force may damage the surface. Thus it has to be considered, especially if the force control performance tolerates a high speed when hitting the object.

\section{A. A One-Dimensional Impact}

According to KICK's law [11], the reaction force between a body and a penetrating impactor under quasi-static, onedimensional conditions is given by

$$
f_{i m p}=c_{i n} \cdot h^{n},
$$

where $c_{i n}$ is a constant factor primarily dominated by the material properties of the contact partners (e.g. their YOUNG's moduli) and the geometry of the impactor [12], [13], [14], $h$ is the indentation depth ${ }^{8}$ and $0<n<\approx 2$ is an exponent often denoted as the work-hardening coefficient. In general there exist two different constants, one for the indentation $c_{i n}$ and one for the rebound phase $c_{r e}$.

To get an impression of the maximum force during an impact the maximum indentation depth $\hat{h}$ according to (13) is needed, which can be found considering the equilibrium of forces at an indentor of mass $M$ in the indention phase, disregarding friction and damping:

$$
M \ddot{h}+f_{i m p}=M \ddot{h}+c_{i n} \cdot h^{n}=0
$$

By solving (14), the dynamics $h(t)$ and $\dot{h}(t)$ of the impact are obtained. The maximum depth $\hat{h}$ is reached at the end

\footnotetext{
${ }^{6}$ In (2) the index $t_{t a}$ is simply denoted by ${ }_{a}$. The signal $s_{a}$ in Fig. 2 can be replaced either by a force or by ${ }^{t a} \mathbf{x}_{t a^{\prime}}$.

${ }^{7}$ The more accurate representation by homogeneous transformation matrices $\mathbf{T}_{t d}(k)=\mathbf{T}_{t a}(k) \cdot{ }^{t a} \mathbf{T}_{t a^{\prime}} \cdot{ }^{t d} \mathbf{T}_{t d^{\prime}}^{-1}$ is independent of a reference system.

${ }^{8}$ According to the previously used notation, $h$ could also be written as ${ }^{o} z_{t a^{\prime}}$, using the coordinate system of Fig. 4 .
} 
of the deceleration, i.e. at $\dot{h}=0$. The remaining plastic indentation depth $h_{\text {rem }}$ will be less than $\hat{h}$, as the deformed surface slightly rebounds due to elasticity. Any sharp edge of the impactor will nonetheless cause plastic deformations. For simplicity we only want to consider the maximum indentation $\hat{h}$.

\section{B. Impact of a Cylindrical Indenter}

According to [15], for a flat faced cylinder (radius $R$ ) colliding normally with a semi-infinite flat surface, the coefficients $n$ and $c_{i n}$ are given by $n=1$ and $c_{i n}=2 R E^{* 9}$, yielding from (14)

$$
M \ddot{h}+2 R E^{*} \cdot h=0 \Rightarrow h(t)=\hat{h} \cdot \sin \left(\sqrt{\frac{2 R E^{*}}{M}} \cdot t\right) .
$$

Using the abbreviation $\omega_{i n}:=\sqrt{\left(2 R E^{*}\right) / M}$, the maximal indentation $\hat{h}$ for a cylindrical punch can be found using the initial velocity of the indentor $\dot{h}(0)=v_{0}$.

$$
\hat{h}=h\left(t=\tau_{i n}\right)=\frac{v_{0}}{\omega_{i n}}
$$

It is important to mention that this solution is valid only until the first total halt of the indentor, i.e. for $0 \leq t \leq \tau_{i n}$. $\tau_{i n}$ is found solving $\dot{h}\left(t=\tau_{i n}\right)=0$, directly yielding $\tau_{i n}=$ $\pi /\left(2 \omega_{i n}\right)$.

The maximum force $\hat{f}_{i m p, c y l}$ can now easily be found by reinserting $\hat{h}$ into (13):

$$
\hat{f}_{i m p, c y l}=v_{0} \cdot \sqrt{2 R E^{*} M}
$$

The total time of the impact, also denoted as the characteristic impact time, $\tau_{\text {cit }}$ consists of the indentation time $\tau_{\text {in }}$ and the rebound time $\tau_{r e}$, which can be found accordingly to $\tau_{i n}$, using $c_{r e}$ instead of $c_{i n}$. If $c_{r e}$ is unknown, which is often the case, a first estimate of $\tau_{c i t}$ can simply be done by

$$
\tau_{c i t} \approx 2 \cdot \tau_{\text {in }}=\frac{\pi}{\omega_{\text {in }}} \neq f\left(v_{0}\right)
$$

implying that both $\tau_{\text {in }}$ and $\tau_{\text {out }}$ are of the same order of magnitude. A remarkable fact is that the characteristic impact time $\tau_{c i t}$ is not a function of the initial velocity, but only of $\omega_{\text {in }}$.

It has to be noted that all given equations only demonstrate the fundamental mechanisms using the simple example of a flat punch. The governing equations (13) and (14) remain valid for other kinds of impactors, but the determination of the coefficients $c_{i n / r e}$ and $n$ becomes complex for other geometries or even in the case with more than a single degree of freedom.

\section{Numerical Results}

In order to assess the influence of the impact for the setup of Fig. 1 and for the experiments of Section V (Setup according to Fig. 6) we use the parameters of Table I.

\footnotetext{
${ }^{9}$ The effective YOUNG's modulus is given by $E^{*}=$ $\left[\frac{1-\nu_{1}^{2}}{E_{1}}+\frac{1-\nu_{2}^{2}}{E_{2}}\right]^{-1}$, where $\nu_{i}$ and $E_{i}$ refer to the POISSON's ratio and the YOUNG's modulus of each contact partner.
}

TABLE I

PARAMETERS USED FOR THE ASSESSMENT OF THE IMPACT

\begin{tabular}{|l|c|c|}
\hline Parameter & Setup of Fig. 1 & Setup of Fig. 6 \\
\hline$R$ & $10 \mathrm{~mm}$ & $8 \mathrm{~mm}$ \\
\hline$M$ & $100 \mathrm{~kg}$ & $4 \mathrm{~kg}$ \\
\hline$E^{*}$ (steel on steel) & $100 \mathrm{GPa}$ & \\
\hline$E^{*}$ (steel on wood) & & $1 \mathrm{GPa} 10$ \\
\hline$v_{0}$ & $20 \mathrm{~mm} / \mathrm{s}$ & $20 \mathrm{~mm} / \mathrm{s}$ \\
\hline
\end{tabular}

According to (18) this yields a characteristic impact time $\tau_{c i t}$ of about $0.7 \mathrm{~ms}$ resp. $1.5 \mathrm{~ms}$ for the two configurations. This is smaller than the sampling time, making it hard to control the impact force.

The maximal impact force $\hat{f}_{i m p, c y l}$ is proportional to the initial velocity. When using the parameters of Table I, (17) yields $9000 \mathrm{~N}$ or $160 \mathrm{~N}$ for the two configurations. This is much more than the measured control error in Section V. Fortunately the force is much smaller when a non-contact sensor has predicted the collision and hence the impact velocity has been reduced almost to zero.

\section{EXPERIMENTS}

Force control methods can be evaluated best when using a laboratory setup. Therefore a test scenario has been selected (Fig. 6) instead of the configuration of Fig. 1 with the swinging manipulated object.

In the selected setup the robot moves down until a significant force is sensed. This happens at the real object pose which is about $10 \mathrm{~mm}$ before the expected object pose. With the first contact the model is updated and a desired force of $10 \mathrm{~N}$ is regulated at the new position.

In addition, when contact is sensed, the robot instantaneously rotates by about $2.5 \mathrm{mrad}$, in order to preserve the real tool orientation in spite of the contact force.

Fig. 7 shows the measured force when using the proposed approach. The peak of the first sensor values is reduced

\footnotetext{
${ }^{10} \mathrm{E}=0.2 \cdots 1.5 \mathrm{GPa}$ have been found for wood which is compressed perpendicular to the grain. Along the grain the values are $4 \cdots 17 \mathrm{GPa}$ [16].
}

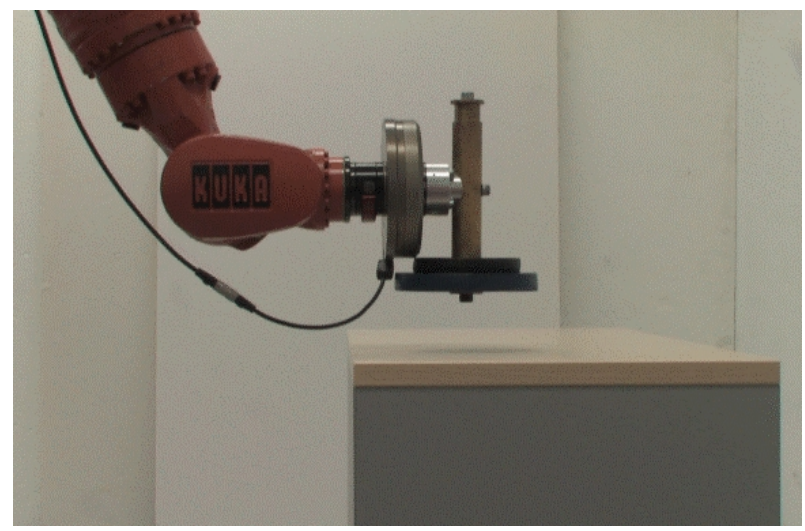

Fig. 6. Set-up for force control, just before the impact. 


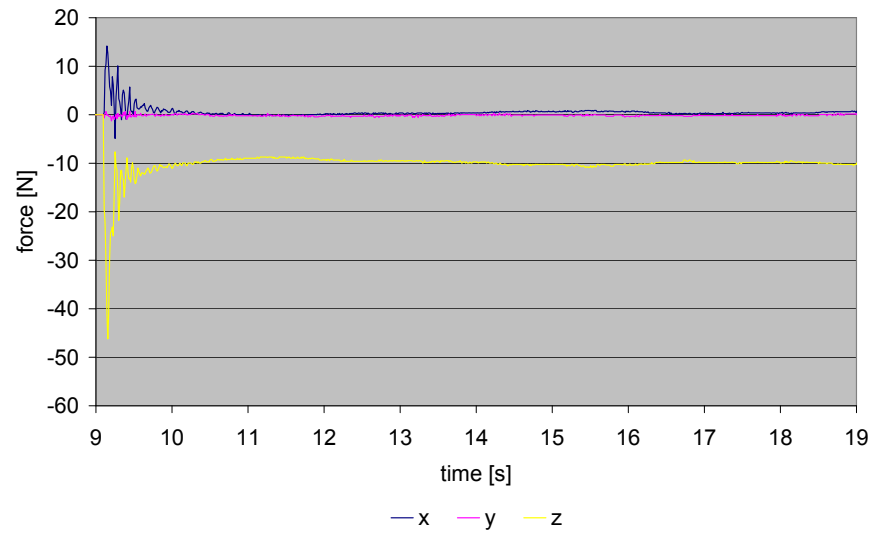

Fig. 7. Measured force during the experiment with the proposed method of section II-B and section III-B.

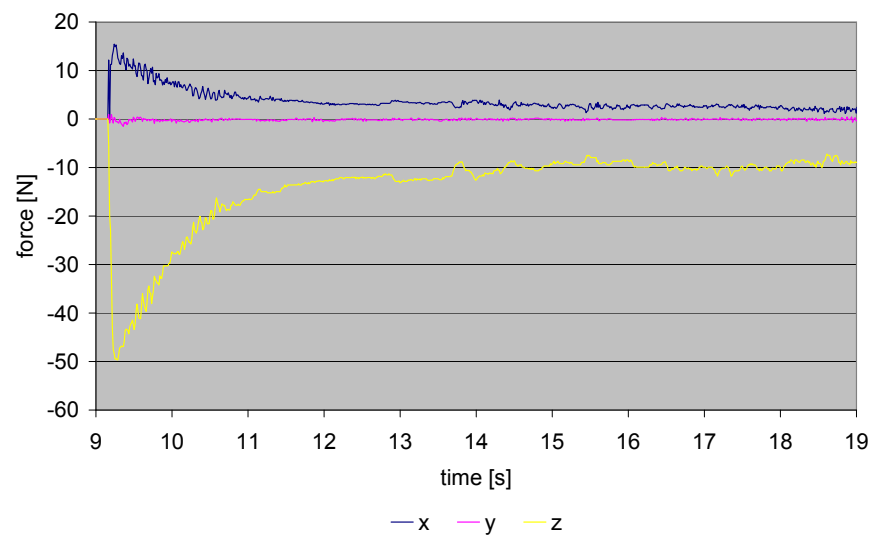

Fig. 8. Measured force during the experiment using the pd-controller of section II-A with the extension of section III-B.

rapidly. A faster reduction is not possible because of the robot dynamics and the characteristics of the feedback controller. Strictly speaking, there is a vibration which is caused by compliance of the robot joints, which is not modeled.

Fig. 8 compares the experiment with the standard approach of (1). In this experiment, in contrast to the classical paradigm, the computation of the desired deflections has been applied, in order to avoid a rotational drift. It can be seen that the settling time is significantly longer. Other controller parameters can improve the first reduction but cause an oscillation later. So the advantage of the presented approach is obvious.

The steady state force depends on the parameters $\mathbf{C}_{p}$ and $\mathbf{C}_{\varphi}$ which may even vary. Therefore an adaptation scheme is used in both experiments, which has not been explained in this paper.

\section{CONClusion AND Future Work}

The paper reviews a force control scheme that allows a fast reaction to unexpected forces that can be explained by a collision of the robot tool with a fixed rigid object. But, besides the measured force, an additional impact force is significant, which cannot be controlled. The method is generalized to typical setups in which the sensor is located distantly from the contact point.

Future work will concentrate on the effect of a compliant sensor during free motion. Oscillation damping is fundamental in this case. In addition, the deflected actual pose of the tcp has to be controlled, not the pose with ignored displacement (=nominal actual pose). This is fundamental during acceleration phases.

In addition, it seems to be useful to consider the compliances in the robot gears since they might be the reason for the vibration in Fig. 7. As the proposed force control scheme is so fast, it excites effects that usually may be neglected.

\section{REFERENCES}

[1] J. Grewe, G. Hirzinger, R. R. Koeppe, C. Strobl, and B. Willberg. Compliant-force-torque-sensor. In Proc. 10th Int. Workshop on Robotics in Alpe-Adria-Danube Region, Vienna, Austria, May 2001.

[2] Schunk GmbH \& Co KG. SCHUNK - Workholding Solutions, Automation Components, Toolholding Components, Gripping Systems, Linear Systems. http://www.schunk.com/ schunk/schunk_websites/products/products_ level_3/product_level3.html?product_level_ $3=296$ \&product_level_2 252 \&product_level_1= 244 \& count $r y=I N T \& 1$ ngCode $=E N \&$ IngCode $2=E N, 2011$.

[3] F. Lange, B. Willberg, and G. Hirzinger. Control of large forces and torques using an asymmetrically arranged compliant sensor. In Proc. Joint 4lth Int. Symp. on Robotics and 6th German Conf. on Robotics ISR/ROBOTIK 2010, Munich, Germany, June 2010.

[4] Mario Prats, Philippe Martinet, Angel P. del Pobil, and Sukhan Lee. Vision/force control in task-oriented grasping and manipulation. In Proc. 2007 IEEE/RSJ Int. Conf. on Intelligent Robots and Systems (IROS), pages 1320-1325, San Diego, CA, USA, 2007.

[5] Paul Hebert, Nicolas Hudson, Jeremy Ma, and Joel Burdick. Fusion of stereo vision, force-torque, and joint sensors for estimation of inhand object location. In Proc. 2011 IEEE Int. Conf. on Robotics and Automation (ICRA), pages 5935-5941, Shanghai, China, 2011.

[6] Brian Mayton, Louis LeGrand, and Joshua R. Smith. An electric field pretouch system for grasping and co-manipulation. In Proc. 2010 IEEE Int. Conf. on Robotics and Automation (ICRA), pages 831-838, Anchorage, AK, USA, May 2010.

[7] A. Kamel, F. Lange, and G. Hirzinger. New aspects of input shaping control to damp oscillations of a compliant force sensor. In Proc. 2008 IEEE Int. Conf. on Robotics and Automation (ICRA), pages 26292635, Pasadena, CA, May 2008.

[8] Luigi Villani and Joris de Schutter. Force control. In Handbook of Robotics, pages 161-185. Springer, 2008.

[9] Joris de Schutter, Herman Bruyninckx, Stefan Dutré, Jan de Geeter, Jayantha Katupitiya, Sabine Demey, and Tine Lefebvre. Estimating first-order geometric parameters and monitoring contact transitions during force-controlled compliant motion. The International Journal of Robotics Research, 18:1161-1184, 1999.

[10] Tine Lefebvre, Herman Bruyninckx, and Joris De Schutter. Polyhedral contact formation identification for autonomous compliant motion: Exact nonlinear bayesian filtering. IEEE Transactions on Robotics, 21(1), Feb 2005.

[11] Friedrich Kick. Das Gesetz der proportionalen Widerstände und seine Anwendungen. A. Felix, Leipzig, 1885.

[12] E.W. Andrews, A.E. Giannakopoulos, E. Plisson, and S. Suresh. Analysis of the impact of a sharp indenter. International Journal of Solids and Structures, 39:281-295, 2002.

[13] A. E. Giannakopoulos and S. Suresh. Determination of elastoplastic properties by instrumented sharp indentation. Scripta materialia, 40:1191-1198, 1999.

[14] M. T. Attaf. Connection between the loading curve models in elastoplastic indentation. Materials Letters, 58:3491-3498, 2004.

[15] I. Sneddon. The relation between load and penetration in the axisymmetric boussinesq problem for a punch of arbitrary profile. International Journal of Engineering Science, 3:47-57, 1965.

[16] M. F. Ashby and D. R. H. Jones. Engineering materials 2: An Introduction to microstructures, processing, and design. ButterworthHeinemann, second edition, 1998. 